\title{
Heavy boson production through the collision of an ultrahigh-energy neutrino on a target nucleon
}

\author{
R.M. García-Hidalgo and A. Rosado \\ Instituto de Física, Universidad Autónoma de Puebla. \\ Apartado Postal J-48, Colonia San Manuel, Puebla, Puebla. 72570, México
}

(January 19, 2019)

\begin{abstract}
We discuss $W$ and $Z$ production through the deep inelastic $\nu_{l} \mathcal{N}$ - scattering in the context of the standard model $S U(3)_{C} \times S U(2)_{L} \times U(1)$ of the strong and electroweak interactions. We find the cross section rates for the process $\nu_{l}+\mathcal{N} \rightarrow l^{-}+W^{+}+X$ for the case of ultrahigh-energy neutrinos $\left(10^{14} \mathrm{eV} \leq\right.$ $\left.E_{\nu} \leq 10^{17} \mathrm{eV}\right)$ colliding on a target nucleon $\left(E_{\mathcal{N}}=m_{\mathcal{N}}\right)$. We also calculate $\sigma\left(\nu_{l}+\mathcal{N} \rightarrow l^{-}+X\right)$ in order to compare it with $\sigma\left(\nu_{l}+\mathcal{N} \rightarrow l^{-}+W^{+}+X\right)$. 13.15.+g, 13.85.Tp, 25.30.Pt
\end{abstract}

Typeset using REVTEX 


\section{INTRODUCTION}

Large-scale neutrino telescopes [1] have as a main goal the detection of ultrahigh-energy (UHE) cosmic neutrinos $\left(E_{\nu} \geq 10^{12} \mathrm{eV}\right)$ produced outside the atmosphere (neutrinos produced by galactic cosmic rays interacting with interstellar gas, and extragalactic neutrinos) [2]. UHE neutrinos can be detected by observing long-range muons produced in chargedcurrent neutrino-nucleon interactions. A very enlightening discussion on UHE neutrino interactions is given by R. Gandhi et al. [3].

The detection of UHE neutrinos will provide us with the possibility to observe $\nu \mathcal{N}$ collisions with a neutrino energy in the range $10^{12} \mathrm{eV} \leq E_{\nu} \leq 10^{21} \mathrm{eV}$ and a target nucleon at rest. Earlier estimations of the $W$ production rates in $\nu \mathcal{N}$-scattering were given in 1970, by H. H. Chen [4], and by J. Reiff [5], in 1971 by R. W. Brown and J. Smith [6], and by F. A. Berends and G. B. West [7]. In those works the calculations were made using neither the standard model [8] nor the parton model [9]. One of the aims of detecting UHE neutrinos is to look for physics beyond [10] the standard model [8]. However, the event rates for most of the exotic processes could be of the same order as the event rates for heavy gauge boson production in $\nu \mathcal{N}$-scattering. Hence, even though the cross sections for heavy boson production are expected not to be large enough to allow for detailed investigations of the heavy bosons properties, it is necessary to calculate them. Only if heavy boson production, which could be an important background for physics beyond the standard model, is completely known and subtracted from the exotic event rates, the new physics could be investigated. Therefore, we discuss in this paper heavy vector boson production in the deep inelastic $\nu_{l} \mathcal{N}$-scattering within the frame of the standard model $S U(3)_{C} \times S U(2)_{L} \times U(1)$ of the strong and electroweak interactions and using the parton model [9] with the parton distribution functions reported by J. Pumplin et al. [11]. We use the CTEQ PDFs provided in a $n_{f}=5$ active flavours scheme.

The cross section for the process $\nu_{l}+\mathcal{N} \rightarrow l^{-}+W^{+}+X$ is expected to be the most important one because it gets contributions from photon exchange diagrams (see figs. 2.1- 
2.5). We thus present results for the total cross section for $W^{+}$production through the processes $\nu_{l}+\mathcal{N} \rightarrow l^{-}+W^{+}+X$ as a function of the neutrino energy in the range $10^{14} \mathrm{eV} \leq$ $E_{\nu} \leq 10^{17} \mathrm{eV}{ }^{1}$ and taking $E_{\mathcal{N}}=m_{\mathcal{N}}$ for the nucleon. We also calculate $\sigma\left(\nu_{l}+\mathcal{N} \rightarrow l^{-}+X\right)$ to find out how it compares to $\sigma\left(\nu_{l}+\mathcal{N} \rightarrow l^{-}+W^{+}+X\right)$.

This paper is organized as follows. In section II, we provide the expressions for the kinematics of the production of a massive vector bosons in deep inelastic neutrino-nucleon scattering and discuss the influence of setting cuts on the phase space of the produced particles. In section III, we perform the explicit calculation of the matrix elements and the differential cross section for polarized scattering. We present and discuss our results for the total cross section for the case of $W^{+}$production in section IV. Finally, section V contains a summary of our conclusions.

\section{KINEMATICS}

In this section we will discuss the kinematics and phase space of the production of a heavy vector boson $B=\left(W^{ \pm}, Z^{0}\right)$ through the inclusive processes

$$
\nu_{l}+\mathcal{N} \rightarrow l^{\prime}+B+X
$$

where $\nu_{l}, \mathcal{N}, l^{\prime}$ and $B$ stand for the incoming neutrino, the target nucleon, the final lepton $\left(\nu_{l}\right.$ or $\left.l^{-}\right)$and the produced massive boson, respectively. We will denote the four-momenta of these particles by $p, P_{\mathcal{N}}, p^{\prime}$ and $k$, respectively. In accordance with the kinematics for the collision of a neutrino on a target nucleon, the following construction is chosen:

$$
p^{\mu}=E_{\nu}(1,0,0,1), \quad P_{\mathcal{N}}^{\mu}=m_{\mathcal{N}}(1,0,0,0) .
$$

As usual we define the invariants:

\footnotetext{
${ }^{1}$ We take $E_{\nu} \leq 10^{17} \mathrm{eV}$, in order to use the CTEQ PDFs in the range of the $\left(x^{\prime}, Q^{\prime 2}\right)$ parameter space in which these have been found to be valid: $10^{-6} \leq x^{\prime} \leq 1$ and $1.3 \mathrm{GeV} \leq \sqrt{Q^{\prime 2}} \leq 10^{4} \mathrm{GeV}$
} 


$$
\begin{aligned}
s & =\left(p+P_{\mathcal{N}}\right)^{2} \\
Q^{2} & =-\left(p-p^{\prime}\right)^{2} \\
\nu & =P_{\mathcal{N}}\left(p-p^{\prime}\right) \\
s^{\prime} & =\left(p+P_{\mathcal{N}}-k\right)^{2} \\
Q^{\prime 2} & =-\left(p-p^{\prime}-k\right)^{2} \\
\nu^{\prime} & =P_{\mathcal{N}}\left(p-p^{\prime}-k\right)
\end{aligned}
$$

and the dimensionless variables:

$$
x=\frac{Q^{2}}{2 \nu}, \quad y=\frac{2 \nu}{s}, \quad \tau=\frac{s^{\prime}}{s}, \quad x^{\prime}=\frac{Q^{\prime 2}}{2 \nu^{\prime}}, \quad y^{\prime}=\frac{2 \nu^{\prime}}{s} .
$$

The physical region of these kinematical variables is obtained by requiring that the scalar products of any two particle four-momenta be positive, the determinants $\Delta_{3}$ of any three particle four-momenta positive, and the determinant $\Delta_{4}$ of the four independent four-momenta (whenever possible we will neglect the nucleon and lepton masses):

$$
\Delta_{4}\left(p, p^{\prime}, P_{\mathcal{N}}, k\right)=\left|\begin{array}{cccc}
0 & p p^{\prime} & p P_{\mathcal{N}} & p k \\
p^{\prime} p & 0 & p^{\prime} P_{\mathcal{N}} & p^{\prime} k \\
P_{\mathcal{N} p} & P_{\mathcal{N}} p^{\prime} & 0 & P_{\mathcal{N}} k \\
k p & k p^{\prime} & k P_{\mathcal{N}} & M_{B}^{2}
\end{array}\right|
$$

negative [12].

From the positivity of the scalar products we find:

$$
0 \leq x<x^{\prime} \leq 1, \quad 0 \leq y^{\prime}<y \leq 1
$$

Explicit evaluation of $\Delta_{4}$ using (3), (4) and (5) gives:

$$
\Delta_{4}=-(s / 2)^{4} x^{2} y^{2}\left(\tau_{b}-\tau\right)\left(\tau-\tau_{a}\right)
$$

where

$$
\begin{aligned}
\tau_{a, b}= & \left(1-x^{\prime}\right)\left(1+y^{\prime}-y\right)+(1 / y)\left\{(1-y)\left[\left(x^{\prime}-x\right)\left(y-y^{\prime}\right)-\mu\right]+x y^{\prime}\right. \\
& \left.\mp 2 \sqrt{x y^{\prime}(1-y)\left[\left(x^{\prime}-x\right)\left(y-y^{\prime}\right)-\mu\right]}\right\} .
\end{aligned}
$$


with $\mu=M_{B}^{2} / s$ and $s=2 m_{\mathcal{N}} E_{\nu}$. The condition $\Delta_{4} \leq 0$ can be used to restrict $\tau$ :

$$
\tau_{a} \leq \tau \leq \tau_{b}
$$

From the requirement that $\tau_{a, b}$ be real, together with $x \geq 0, y^{\prime} \geq 0$ and $y \leq 1$, there follows the inequality

$$
\left(x^{\prime}-x\right)\left(y-y^{\prime}\right) \geq \mu
$$

which is equivalent to $\Delta_{3}\left(p, p^{\prime}, k\right) \geq 0$. Inequality (10) can be used to replace (6) by the more stringent relations:

$$
\begin{array}{lr}
0 \leq x \leq 1-\mu / y, & x+\mu / y \leq x^{\prime} \leq 1, \\
\mu \leq y \leq 1, & 0 \leq y^{\prime}<y-\mu /\left(x-x^{\prime}\right) .
\end{array}
$$

The expressions given in (8), (9) and (11) define the physical region for the dimensionless variables $x, x^{\prime}, y, y^{\prime}$ and $\tau$. We have taken all fermion masses to be zero, this implies that in the calculation of the total cross section the integration over the momentum transfer square extends up to zero. Hence, we have no natural regulator for the contribution of the photon-exchange diagrams. This is one of the reasons why we need cuts on $Q^{2}$ and $Q^{\prime 2}$. Another reason is that the parton distributions can be used only when $Q^{2}$ and $Q^{\prime 2}$ are not too small. Furthermore, in order to separate deep inelastic from elastic scattering, a cut on the invariant mass $W$ of the unobserved particles in the final state is required. Therefore, besides the kinematical conditions (8), (9), (11) we have in general also the following:

$$
\begin{aligned}
& Q^{2}=s x y \geq Q_{c}^{2}, \\
& Q^{\prime 2}=s x^{\prime} y^{\prime} \geq Q_{c}^{\prime 2}, \\
& W=s y^{\prime}\left(1-x^{\prime}\right)+m_{\mathcal{N}}^{2} \geq W_{c} .
\end{aligned}
$$

The cuts for $Q^{2}, Q^{\prime 2}$ and $W$ constrain further the physically allowed region for the process (1). This region can now be written in terms of the dimensionless variables as follows:

$$
\begin{array}{cc}
y_{a} \leq y \leq y_{b}, & x_{a} \leq x \leq x_{b}, \\
x_{a}^{\prime} \leq x^{\prime} \leq x_{b}^{\prime}, & y_{a}^{\prime} \leq y^{\prime} \leq y_{b}^{\prime} .
\end{array}
$$


with

$$
\begin{aligned}
& y_{a}=\max \left\{\left(M_{B}+\sqrt{W_{c}}\right)^{2} / s,\left(M_{B}^{2}+Q_{c}^{2}\right) / s\right\}, \quad y_{b}=1, \\
& x_{a}=Q_{c}^{2} / s y, \quad x_{b}=1-\left(M_{B}+\sqrt{W_{c}}\right)^{2} / s y, \\
& x_{a}^{\prime}=\max \left\{\frac{1}{2 y}\left[y(1+x)+\left(M_{B}^{2}-W_{c}\right) / s-\sqrt{\left[y(1-x)-\left(M_{B}^{2}+W_{c}\right) / s\right]^{2}-4 M_{B}^{2} W_{c} / s^{2}}\right],\right. \\
&\left.\frac{1}{2 y}\left[x y+\left(M_{B}^{2}+Q_{c}^{\prime 2}\right) / s+\sqrt{\left[x y+\left(M_{B}^{2}+Q_{c}^{\prime 2}\right) / s\right]^{2}-4 x y Q_{c}^{\prime 2} / s}\right]\right\}, \\
& x_{b}^{\prime}=\frac{1}{2 y}\left\{y(1+x)+\left(M_{B}^{2}-W_{c}\right) / s+\sqrt{\left[y(1-x)-\left(M_{B}^{2}+W_{c}\right) / s\right]^{2}-4 M_{B}^{2} W_{c} / s^{2}}\right\}, \\
& y_{a}^{\prime}=\max \left\{W_{c} / s\left(1-x^{\prime}\right), Q_{c}^{\prime 2} / s x^{\prime}\right\}, \quad y_{b}^{\prime}=y-M_{B}^{2} / s\left(x^{\prime}-x\right) .
\end{aligned}
$$

The limits for $\tau$ are not modified by the cuts given in (12), thus remaining as in (9).

\section{THE DIFFERENTIAL CROSS SECTION FOR INCLUSIVE BOSON PRODUCTION}

The differential cross section $d \sigma^{\nu_{l} \mathcal{N}}$ for (1) is calculated in the parton model from the cross section $d \sigma^{\nu_{l} q}$ of the parton subprocess

$$
\nu_{l}+q \rightarrow l^{\prime}+q^{\prime}+B
$$

(where $\nu_{l}, q, l^{\prime}, q^{\prime}$ and $B$ stand for the incoming neutrino, a quark inside the target nucleon, the final lepton $\left(\nu_{l}\right.$ or $\left.l^{-}\right)$, the outgoing quark and the produced massive boson) and the parton distribution functions $f_{q}\left(x^{*}, \tilde{Q}^{2}\right)$, which are the probabilities to find a quark $q$ with the fraction $x^{*}$ of the nucleon momentum: $q^{\mu}=x^{*} P_{\mathcal{N}}^{\mu}$, in a scattering process with momentum transfer square $\tilde{Q}^{2}$. We will denote the four-momenta of these particles by $p, q, p^{\prime}, q^{\prime}$ and $k$, respectively. As usual, we define for the parton process the invariant variables $\hat{s}, \hat{Q}^{2}, \ldots, \hat{\nu}^{\prime}$ and using the definitions given in (3) we get the following relations: 


$$
\begin{array}{ll}
\hat{s}=(p+q)^{2} & =x^{*} s, \\
\hat{Q}^{2}=-\left(p-p^{\prime}\right)^{2} & =Q^{2}, \\
\hat{\nu}=q\left(p-p^{\prime}\right) & =x^{*} \nu, \\
\hat{s}^{\prime}=(p+q-k)^{2} & =s^{\prime}-\left(1-x^{*}\right) s+2\left(1-x^{*}\right)\left(\nu-\nu^{\prime}\right), \\
\hat{Q}^{\prime 2}=-\left(p-p^{\prime}-k\right)^{2} & =Q^{\prime 2}, \\
\hat{\nu}^{\prime}=q\left(p-p^{\prime}-k\right) & =x^{*} \nu^{\prime},
\end{array}
$$

The variables $\hat{s}, \ldots, \hat{\nu}^{\prime}$ are not independent. For massless partons we have $\hat{Q}^{\prime 2}=2 \hat{\nu}^{\prime}$ and consequently $x^{*}=Q^{\prime 2} / 2 \nu^{\prime}$. Comparing with the definition of $x^{\prime}$ given in (4) we conclude that $x^{\prime}=x^{*}$, i. e. $q^{\mu}=x^{\prime} P_{\mathcal{N}}^{\mu}$.

The parton cross section is obtained from the invariant matrix element $\mathcal{M}\left(\nu_{l} q \rightarrow l^{\prime} q^{\prime} B\right)$ :

$$
d \sigma^{\nu_{l} q}=\frac{(2 \pi)^{-5}}{2 \hat{s}} \frac{1}{4} \sum_{s, \lambda}\left|\mathcal{M}^{\nu_{l} q}\right|^{2} d \Gamma_{3} .
$$

The sum is performed over all fermion spin states $s$ and the polarizations $\lambda$ of the produced boson. With help of the different sets of variables introduced in (15), (3) and (4), and by using (7), the 3 -particle phase space $d \Gamma_{3}$ can be expressed as follows:

$$
\begin{aligned}
d \Gamma_{3} & =\frac{d^{3} p^{\prime}}{2 E_{p^{\prime}}} \frac{d^{3} q^{\prime}}{2 E_{q^{\prime}}} \frac{d^{3} k^{\prime}}{2 E_{k}} \delta^{4}\left(p+q-p^{\prime}-q^{\prime}-k\right) \\
& =\frac{\pi}{8 \hat{s}} \frac{d \hat{Q}^{2} d \hat{\nu} d \hat{s}^{\prime} d \hat{Q}^{\prime 2} d \hat{\nu}^{\prime}}{\sqrt{-\Delta_{4}\left(p, q, p^{\prime}, k\right)}} \delta\left(\hat{Q}^{\prime 2}-2 \hat{\nu}^{\prime}\right) \\
& =\frac{\pi}{8 s} \frac{d Q^{2} d \nu d s^{\prime} d Q^{\prime 2} d \nu^{\prime}}{\sqrt{-\Delta_{4}\left(p, P_{\mathcal{N}}, p^{\prime}, k\right)}} \delta\left(Q^{\prime 2}-2 x^{\prime} \nu^{\prime}\right) \\
& =\frac{\pi s}{8} \frac{d x d y d y^{\prime} d \tau}{\sqrt{\left(\tau_{b}-\tau\right)\left(\tau-\tau_{a}\right)}}
\end{aligned}
$$

From (16) and (17) we obtain

$$
d \sigma^{\nu_{l} q}=\frac{1}{2^{7}(2 \pi)^{4}} \sum_{s, \lambda}\left|\mathcal{M}^{\nu_{l} q}\right|^{2} \frac{1}{x^{\prime}} \frac{d x d y d y^{\prime} d \tau}{\sqrt{\left(\tau_{b}-\tau\right)\left(\tau-\tau_{a}\right)}}
$$

The Feynman diagrams for $\mathcal{M}^{\nu_{l} q}$ are depicted in fig. 1. The heavy boson can be produced from the lepton line (fig. 1: a, b), the (anti)quark line (fig. 1: c, d) or via the non-Abelian vertex from the line of the exchanged bosons (fig. 1: e). We write

$$
\mathcal{M}^{\nu_{l} q}=\mathcal{M}^{\nu_{l} q, l}+\mathcal{M}^{\nu_{l} q, h}+\mathcal{M}^{\nu_{l} q, n}
$$


Diagrams containing the exchange of Higgs bosons have been neglected because of the smallness of the Higgs fermion coupling. We present the explicit expressions for these matrix elements in a way which is suited not only for the standard model of the electroweak interaction but also for extended models containing more vector bosons than the SM. Therefore, denoting with $f$ the fermions and with $B$ the gauge bosons, we define the couplings:

$$
\bar{\psi}_{f^{\prime}} \psi_{f} B_{\mu} \Rightarrow i \gamma_{\mu}\left(v_{f^{\prime} f}^{B}-a_{f^{\prime} f}^{B} \gamma_{5}\right)=i \gamma_{\mu}\left(L_{f^{\prime} f}^{B} P_{L}+R_{f^{\prime} f}^{B} P_{R}\right)
$$

and

$$
B_{\mu}(p) B_{\nu}^{\prime}\left(p^{\prime}\right) B_{\rho}^{\prime \prime}\left(p^{\prime \prime}\right) \Rightarrow i g_{B B^{\prime} B^{\prime \prime}}\left\{g_{\mu \nu}\left(p-p^{\prime}\right)_{\rho}+g_{\nu \rho}\left(p^{\prime}-p^{\prime \prime}\right)_{\mu}+g_{\mu \rho}\left(p^{\prime \prime}-p\right)_{\nu}\right\}
$$

with $P_{L, R}=\left(1 \mp \gamma_{5}\right) / 2, \quad L_{f^{\prime} f}^{B}=v_{f^{\prime} f}^{B}+a_{f^{\prime} f}^{B}$, and $R_{f^{\prime} f}^{B}=v_{f^{\prime} f}^{B}-a_{f^{\prime} f}^{B}$.

In the case of the standard model the couplings of the fermions to the $Z$ and $W$ bosons are given as follows $(e=\sqrt{4 \pi \alpha})$ :

$$
\begin{aligned}
& v_{f^{\prime} f}^{Z}=-e\left(T_{f}^{3}-2 Q_{f} \sin ^{2} \theta_{W}\right) / \sin 2 \theta_{W}, \\
& a_{f^{\prime} f}^{Z}=-e T_{f}^{3} / \sin 2 \theta_{W}, \\
& v_{f^{\prime} f}^{W}=-e /\left(2 \sqrt{2} \sin \theta_{W}\right), \\
& a_{f^{\prime} f}^{W}=-e /\left(2 \sqrt{2} \sin \theta_{W}\right) .
\end{aligned}
$$

$Q_{f}$ and $T_{f}^{3}$ stand for the charge and the third component of the isospin of the fermion $\mathrm{f}$. For the three bosons non-Abelian couplings we have:

$$
\begin{aligned}
g_{\gamma W^{+} W^{-}} & =e, \\
g_{Z^{0} W^{+} W^{-}} & =e \cos \theta_{W} / \sin \theta_{W} .
\end{aligned}
$$

The invariant matrix elements $\mathcal{M}^{\nu_{l} q, a}, a=\{l, h, n\}$ for the production of the boson $B$ (polarization vector $\left.\varepsilon^{\mu}(k, \lambda)\right)$ in the scattering of the neutrino with initial and the outgoing lepton with final longitudinal polarization $P_{l}, P_{l^{\prime}}$ and the quark with initial and final polarizations $P_{q}, P_{q^{\prime}}$ can be expressed, taking all fermion masses to be zero (hence $P_{l^{\prime}}=P_{l}$ and $P_{q^{\prime}}=P_{q}$ ) in the following form

$$
\mathcal{M}_{P_{q}, P_{l}}^{\nu_{l} q, a}=J_{\mu}^{h, P_{q}} f_{P_{q}, P_{l}}^{r, a} \mathcal{F}_{r}^{\mu \nu} J_{\nu}^{l, P_{l}}
$$


where the left-, right-handed currents of the leptons and quarks are defined as usual

$$
J_{\mu}^{l, P_{l}}=\bar{u}\left(p^{\prime}\right) \gamma_{\mu} P_{l} u(p)
$$

and

$$
J_{\mu}^{h, P_{q}}=\bar{u}\left(q^{\prime}\right) \gamma_{\mu} P_{q} u(p)
$$

and the tensors $\mathcal{F}_{r}^{\mu \nu}, r=\{1,2,3\}$ are defined as follows:

$$
\mathcal{F}_{1}^{\mu \nu}=g^{\mu \nu}, \quad \mathcal{F}_{2}^{\mu \nu}=\varepsilon^{\mu} k^{\nu}-k^{\mu} \varepsilon^{\nu}, \quad \mathcal{F}_{3}^{\mu \nu}=i \varepsilon^{\mu \nu \rho \sigma} \varepsilon_{\rho} k_{\sigma} .
$$

The invariant functions $f_{P_{q}, P_{l}}^{r, a}$ are built from the propagators and couplings in the form:

$$
\begin{array}{ll}
f_{P_{q}, P_{l}}^{1, l}=2 \varepsilon p C_{P_{q}, P_{l}}^{l, i}+2 \varepsilon p^{\prime} C_{P_{q}, P_{l}}^{l, f}, & f_{P_{q}, P_{l}}^{2, l}=C_{P_{q}, P_{l}}^{l, i}-C_{P_{q}, P_{l}}^{l, f}, \\
f_{P_{q}, P_{l}}^{3, l}=(-1)^{S_{1}}\left(C_{P_{q}, P_{l}}^{l, i}+C_{P_{q}, P_{l}}^{l, f}\right), & \\
f_{P_{q}, P_{l}}^{1, h}=2 \varepsilon q C_{P_{q}, P_{l}}^{h, i}+2 \varepsilon q^{\prime} C_{P_{q}, P_{l}}^{h, f}, & f_{P_{q}, P_{l}}^{2, h}=C_{P_{q}, P_{l}}^{h, i}-C_{P_{q}, P_{l}}^{h, f}, \\
f_{P_{q}, P_{l}}^{3, h}=(-1)^{S_{2}}\left(C_{P_{q}, P_{l}}^{h, i}+C_{P_{q}, P_{l}}^{h, f}\right), & \\
f_{P_{q}, P_{l}}^{1, n}=2 \varepsilon\left(p-p^{\prime}\right) C_{P_{q}, P_{l}}^{n, i}, & f_{P_{q}, P_{l}}^{2, n}=-C_{P_{q}, P_{l}}^{n}, \\
f_{P_{q}, P_{l}}^{3, n}=0, &
\end{array}
$$

with $S_{1}=0,1,0,1$ and $S_{2}=0,0,1,1$ for $P_{q} P_{l}=L L, L R, R L, R R$, respectively, and

$$
\begin{aligned}
& C_{L, L}^{l, i}=\sum_{B^{\prime}, l^{\prime \prime}} L_{l^{\prime} l^{\prime \prime}}^{B^{\prime}} L_{l^{\prime \prime} \nu_{l}}^{B} L_{q^{\prime} q}^{B^{\prime}} /\left[\left(Q^{\prime 2}+M_{B^{\prime}}^{2}\right)\left(M_{B}^{2}-2 k p\right)\right] \\
& C_{L, L}^{l, f}=\sum_{B^{\prime}, l^{\prime \prime}} L_{l^{\prime} l^{\prime \prime}}^{B} L_{l^{\prime \prime} \nu_{l}}^{B^{\prime}} L_{q^{\prime} q}^{B^{\prime}} /\left[\left(Q^{\prime 2}+M_{B^{\prime}}^{2}\right)\left(M_{B}^{2}+2 k p^{\prime}\right)\right] \\
& C_{L, L}^{h, i}=\sum_{B^{\prime}, q^{\prime \prime}} L_{\nu_{l} l^{\prime}}^{B^{\prime}} L_{q^{\prime} q^{\prime \prime}}^{B^{\prime}} L_{q^{\prime \prime} q}^{B} /\left[\left(Q^{2}+M_{B^{\prime}}^{2}\right)\left(M_{B}^{2}-2 k q\right)\right] \\
& C_{L, L}^{h, f}=\sum_{B^{\prime}, q^{\prime \prime}} L_{\nu_{l} l^{\prime}}^{B^{\prime}} L_{q^{\prime} q}^{B} L_{q^{\prime \prime} q}^{B} /\left[\left(Q^{2}+M_{B^{\prime}}^{2}\right)\left(M_{B}^{2}+2 k q^{\prime}\right)\right] \\
& C_{L, L}^{n}=\sum_{B^{\prime}, B^{\prime \prime}} L_{\nu_{l} l^{\prime}}^{B^{\prime}} L_{q^{\prime} q}^{B^{\prime \prime}} g_{B^{\prime} B B^{\prime \prime}} /\left[\left(Q^{2}+M_{B^{\prime}}^{2}\right)\left(Q^{\prime 2}+M_{B^{\prime \prime}}^{2}\right)\right] .
\end{aligned}
$$

for left-handed polarization of leptons and quarks. For other polarizations L has to be replaced by $\mathrm{R}$ in a suitable way in (19).

The next step in the calculation of $d \sigma_{P_{q}, P_{l}}^{l \mathcal{N}}$ is to square $\mathcal{M}_{P_{q}, P_{l}}^{\nu_{l} q}$ : 


$$
\left|\mathcal{M}_{P_{q}, P_{l}}^{\nu_{l} q}\right|^{2}=\left(\sum_{a} \mathcal{M}_{P_{q}, P_{l}}^{\nu_{l} q, a}\right)\left(\sum_{b} \mathcal{M}_{P_{q}, P_{l}}^{\nu_{l} q, b}\right)^{*}
$$

and sum over all fermion spin states $s$ and the polarizations $\lambda$ of the produced boson.

We have

$$
\begin{aligned}
\sum_{s, \lambda} \mathcal{M}_{P_{q}, P_{l}}^{\nu_{l} q, a}\left(\mathcal{M}_{P_{q}, P_{l}}^{\nu_{l} q, b}\right)^{*} & =\sum_{s, \lambda, r, r^{\prime}} J_{\mu}^{h, P_{q}} f_{P_{q}, P_{l}}^{r, a} \mathcal{F}_{r}^{\mu \nu} J_{\nu}^{l, P_{l}}\left(J_{\nu^{\prime}}^{l, P_{l}}\right)^{*} f_{P_{q}, P_{l}}^{r^{\prime}, b}\left(\mathcal{F}_{r^{\prime}}^{\mu^{\prime} \nu^{\prime}}\right)^{*}\left(J_{\mu^{\prime}}^{h, P_{q}}\right)^{*} \\
& =\sum_{\lambda, r, r^{\prime}} \mathcal{H}_{\mu \mu^{\prime}}^{P_{q}} \mathcal{L}_{\nu \nu^{\prime}}^{P_{l}} f_{P_{q}, P_{l}}^{r, a} \mathcal{F}_{r}^{\mu \nu} f_{P_{q}, P_{l}}^{r^{\prime}, b}\left(\mathcal{F}_{r^{\prime}}^{\mu^{\prime} \nu^{\prime}}\right)^{*} \\
& =\sum_{\lambda, r, r^{\prime}} T_{P_{q}, P_{l}}^{r, r^{\prime}} f_{P_{q}, P_{l}}^{r, a} f_{P_{q}, P_{l}}^{r^{\prime}, b}
\end{aligned}
$$

with

$$
\mathcal{H}_{\mu \nu}^{P_{q}}=\mathcal{J}_{\mu}^{h, P_{q}} \mathcal{J}_{\nu}^{h, P_{q}}, \quad \mathcal{L}_{\mu \nu}^{P_{l}}=\mathcal{J}_{\mu}^{l, P_{l}} \mathcal{J}_{\nu}^{l, P_{l}},
$$

and

$$
T_{P_{q}, P_{l}}^{r, r^{\prime}}=\mathcal{H}_{\mu \mu^{\prime}}^{P_{q}} \mathcal{F}_{r}^{\mu \nu}\left(\mathcal{F}_{r^{\prime}}^{\mu^{\prime} \nu^{\prime}}\right)^{*} \mathcal{L}_{\nu \nu^{\prime}}^{P_{l}}
$$

Using the expressions given for $\mathcal{H}_{\mu \nu}^{P_{q}}$ and $\mathcal{L}_{\mu \nu}^{P_{q}}$ in the appendix and the fact that the $f_{P_{q}, P_{l}}^{r, a}, s$ are real functions, it is straightforward to show that

$$
\mathcal{M}_{P_{q}, P_{l}}^{\nu_{l} q, a}\left(\mathcal{M}_{P_{q}, P_{l}}^{\nu_{l} q, b}\right)^{*}=\left(\mathcal{M}_{P_{q}, P_{l}}^{\nu_{l} q, a}\right)^{*} \mathcal{M}_{P_{q}, P_{l}}^{\nu_{l} q, b}
$$

Hence

$$
\begin{aligned}
\sum_{s, \lambda}\left|\mathcal{M}_{P_{q}, P_{l}}^{\nu_{l} q}\right|^{2}= & \sum_{s, \lambda}\left(\left|\mathcal{M}_{P_{q}, P_{l}}^{\nu_{l} q, l}\right|^{2}+\left|\mathcal{M}_{P_{q}, P_{l}}^{\nu_{l} q, h}\right|^{2}+\left|\mathcal{M}_{P_{q}, P_{l}}^{\nu_{l} q, n}\right|^{2}\right) \\
& +2 \operatorname{Re}\left\{\sum_{s, \lambda}\left(\mathcal{M}_{P_{q}, P_{l}}^{\nu_{l} q, l}\left(\mathcal{M}_{P_{q}, P_{l}}^{\nu_{l} q, h}\right)^{*}+\mathcal{M}_{P_{q}, P_{l}}^{\nu_{l} q, l}\left(\mathcal{M}_{P_{q}, P_{l}}^{\nu_{l} q, n}\right)^{*}+\mathcal{M}_{P_{q}, P_{l}}^{\nu_{l} q, h}\left(\mathcal{M}_{P_{q}, P_{l}}^{\nu_{l} q, n}\right)^{*}\right)\right\}
\end{aligned}
$$

Explicit expressions for the quantities $T^{r, r^{\prime}}$ are presented in the appendix. Also, the explicit summation over the polarizations $\lambda$ of the produced boson is presented there.

A heavy weak boson $B$ can be produced in deep inelastic $\nu_{l} \mathcal{N}$ collisions ${ }^{2}$ via the following processes:

\footnotetext{
${ }^{2}$ the results for $\bar{\nu}_{l} \mathcal{N}$-scattering can be obtained from those of $\nu_{l} \mathcal{N}$-scattering by the replacements $\nu_{l} \rightarrow \bar{\nu}_{l}, W^{ \pm} \rightarrow W^{\mp}, l^{-} \rightarrow l^{+}$and $u$-type quarks $\leftrightarrow d$-type quarks
} 


$$
\begin{aligned}
& \nu_{l}+\mathcal{N} \rightarrow \nu_{l}+Z^{0}+X, \\
& \nu_{l}+\mathcal{N} \rightarrow l^{-}+Z^{0}+X, \\
& \nu_{l}+\mathcal{N} \rightarrow \nu_{l}+W^{+}+X, \\
& \nu_{l}+\mathcal{N} \rightarrow \nu_{l}+W^{-}+X, \\
& \nu_{l}+\mathcal{N} \rightarrow l^{-}+W^{+}+X, \\
& \nu_{l}+\mathcal{N} \rightarrow l^{-}+W^{-}+X,
\end{aligned}
$$

The diagrams which contribute in the lowest order at the quark level to the different reaction mechanisms (production at the leptonic vertex, at the hadronic vertex and through the boson self interaction) of all these processes, are depicted in figs. $2.1-2.5$. The process $\nu_{l}+\mathcal{N} \rightarrow l^{-}+W^{-}+X$, is forbidden in the lowest order. We see from these figures that the reactions (P.1) - (P.4) only get contribution from heavy boson exchange diagrams and therefore their total cross sections are expected to be very small and we will not be discussed in this work.

The final step in the evaluation of $d \sigma^{\nu_{l} \mathcal{N}}$ consists now in putting together the parton cross sections $d \sigma^{\nu_{l} q}$ and the parton distribution functions $f_{q}\left(x^{\prime}, \tilde{Q}^{2}\right)$. In contrast to deep inelastic $\nu_{l} \mathcal{N}$-scattering the choice of $\tilde{Q}^{2}$ is not unambiguous in our case since the momentum transfer to the nucleon depends on the reaction mechanism (in other words, whether the boson is emitted at the leptonic or at the hadronic vertex). For leptonic production it is reasonable to take $\tilde{Q}^{2}=-\left(p-p^{\prime}-k\right)^{2}=Q^{\prime 2}$ since $p-p^{\prime}-k$ is the momentum transfer to the nucleon. In the case of hadronic production the obvious choice is $\tilde{Q}^{2}=-\left(p-p^{\prime}\right)^{2}=Q^{2}$. For the non-Abelian diagrams a simple kinematical argument is not sufficient. For heavy boson production in neutrino quark scattering, unitarity would be violated without a coupling between the $W$, $Z$ and $\gamma$ bosons. Therefore unitarity is restored through strong cancellations between these non-Abelian diagrams and either the leptonic or the hadronic contributions to neutrino lepton scattering.

In this work, we will neglect the contribution from heavy boson exchange diagrams. Hence, we calculate the cross section of process $\nu_{l}+\mathcal{N} \rightarrow l^{-}+W^{+}+X$ with the following practical prescription which guarantees the restoration of unitarity 


$$
d \sigma^{\nu_{l} \mathcal{N}}=\sum_{q} \int d x^{\prime} f_{q}\left(x^{\prime}, Q^{\prime 2}\right)\left(d \sigma_{l l}^{\nu_{l} q}+d \sigma_{l n}^{\nu_{l} q}+d \sigma_{n n}^{\nu_{l} q}\right) .
$$

This prescription collects the expressions where the heavy boson is emitted from the lepton line or the non-Abelian vertex and the interference of these reaction mechanisms.

\section{RESULTS FOR $W^{+}$PRODUCTION VIA $\nu \mathcal{N}$ COLLISIONS}

We have already pointed out in the previous section that the process $\nu_{l}+\mathcal{N} \rightarrow l^{-}+W^{+}+$ $X$ is the only one which gets contribution from photon-exchange diagrams. Therefore, we will restrict ourselves to calculate numerically the cross section for $W^{+}$-production through this process, leaving out the contribution from heavy boson exchange diagrams.

We obtain our numerical results using the standard model of the electroweak interactions [8], taking $M_{W}=80.4 \mathrm{GeV}$ for the mass of the charged boson $W$ and $M_{Z}=91.2 \mathrm{GeV}$ for the mass of the neutral boson $Z$ (hence, $\sin ^{2} \theta_{W}=0.223$ for the electroweak mixing angle) [13]. We present results for the case of unpolarized deep inelastic $\nu \mathcal{N}$-scattering with a neutrino energy in the range $10^{14} \mathrm{eV} \leq E_{\nu} \leq 10^{14} \mathrm{eV}$ and the nucleon at rest ${ }^{1}$, i.e. a target nucleon. We take cuts of $4 \mathrm{GeV}^{2}, 4 \mathrm{GeV}^{2}$ and $10 \mathrm{GeV}^{2}$ for $Q^{2}, Q^{\prime 2}$ and the invariant mass $W$, respectively. These values for the cuts are suited for the parton distribution functions

of J. Pumplin et al. [11] which we will use in our calculations. In fig. 3, we show the dependence of the cross section for the reaction $\nu_{l}+P \rightarrow l^{-}+W^{+}+X$ on the cuts on the momenta transfer square $\left(Q^{2}, Q^{\prime 2}\right)$. Taking a cut $W_{c}=10 \mathrm{GeV}^{2}$ for the invariant mass $W$, we plot $\sigma\left(\nu_{l}+P \rightarrow l^{-}+W^{+}+X\right)$ for the following cases: a) $Q_{c}^{2}=Q_{c}^{\prime 2}=2 G e V^{2}$, b) $Q_{c}^{2}=Q_{c}^{\prime 2}=4 \mathrm{GeV}^{2}$, c) $Q_{c}^{2}=Q_{c}^{\prime 2}=6 \mathrm{GeV}^{2}$ and d) $Q_{c}^{2}=Q_{c}^{\prime 2}=8 \mathrm{GeV}^{2}$. We observe in this graph that the total cross section rates of the process $\nu_{l}+\mathcal{N} \rightarrow l^{-}+W^{+}+X$ do not depend strongly on the choice of the cuts on the momenta transfer square $\left(Q^{2}\right.$ and $\left.Q^{\prime 2}\right)$, when they take on values of a few $G e V^{2}$.

In fig. 4 we plot $\sigma\left(\nu_{l}+P \rightarrow l^{-}+W^{+}+X\right)$ and $\sigma\left(\nu_{l}+P \rightarrow l^{-}+X\right)$ as a function of $E_{\nu}$. We obtain $\sigma\left(\nu_{l}+P \rightarrow l^{-}+W^{+}+X\right)=2.3 \times 10^{-35} \mathrm{~cm}^{2}$ for $E_{\nu}=10^{17} \mathrm{eV}$. In fig. 5, we show $\sigma\left(\nu_{l}+N \rightarrow l^{-}+W^{+}+X\right)$ and $\sigma\left(\nu_{l}+N \rightarrow l^{-}+X\right)$ as a function of $E_{\nu}$. We get 
$\sigma\left(\nu_{l}+N \rightarrow l^{-}+W^{+}+X\right)=1.8 \times 10^{-35} \mathrm{~cm}^{2}$ for $E_{\nu}=10^{17} \mathrm{eV}$. Finally, in fig. 6, we present our results for $\sigma\left(\nu_{l}+\mathcal{N} \rightarrow l^{-}+W^{+}+X\right) / \sigma\left(\nu_{l}+\mathcal{N} \rightarrow l^{-}+X\right)(\mathcal{N}: P, N)$ as a function of $E_{\nu}$, with $E_{\mathcal{N}}=m_{\mathcal{N}}$. In particular, we obtain $\sigma\left(\nu_{l}+P \rightarrow l^{-}+W^{+}+X\right) / \sigma\left(\nu_{l}+P \rightarrow l^{-}+X\right)=4.7 \times 10^{-3}$ and $\sigma\left(\nu_{l}+N \rightarrow l^{-}+W^{+}+X\right) / \sigma\left(\nu_{l}+N \rightarrow l^{-}+X\right)=3.6 \times 10^{-3}$ for $E_{\nu}=10^{17} \mathrm{eV}$.

\section{CONCLUSIONS}

We have presented the general formulas for the cross section of the production of massive vector bosons in deep inelastic neutrino nucleon scattering in the framework of the standard model. The expressions for the matrix elements are given in a way that is suitable for extended models containing more vector bosons than the SM.

We have neglected the contribution from heavy boson exchange diagrams in our numerical calculations. Hence, we have given numerical results only for the total cross section rates of the process $\nu_{l}+\mathcal{N} \rightarrow l^{-}+W^{+}+X(\mathcal{N}: P, N$.) because this reaction is the only one which gets contribution from photon-exchange diagrams in the lowest order of $\alpha$. Considering the nucleon at rest, taking $M_{W}=80.4 \mathrm{GeV}, \sin ^{2} \theta_{W}=0.223$, a neutrino energy in the range $10^{14} \mathrm{eV} \leq E_{\nu} \leq 10^{17} \mathrm{eV}$, setting cuts of $4 \mathrm{GeV}^{2}$ and $10 \mathrm{GeV}^{2}$ for the momenta transfer

square $\left(Q^{2}\right.$ and $\left.Q^{\prime 2}\right)$ and the invariant mass $(W)$. We made use of the parton distribution functions of J. Pumplin et al., we used the CTEQ PDFs provided in a $n_{f}=5$ active flavours scheme. In particular, we have obtained $\sigma\left(\nu_{l}+P \rightarrow l^{-}+W^{+}+X\right)=2.3 \times 10^{-35} \mathrm{~cm}^{2}$ and $\sigma\left(\nu_{l}+N \rightarrow l^{-}+W^{+}+X\right)=1.8 \times 10^{-35} \mathrm{~cm}^{2}$ for $E_{\nu}=10^{17} \mathrm{eV}$. We have also shown that the total cross section rates of the process $\nu_{l}+\mathcal{N} \rightarrow l^{-}+W^{+}+X$ do not depend strongly on the choice of the cuts on the momenta transfer square $\left(Q^{2}\right.$ and $\left.Q^{\prime 2}\right)$, when we take them equal to a few $\mathrm{GeV}^{2}$.

We have also presented results for $\sigma\left(\nu_{l}+\mathcal{N} \rightarrow l^{-}+W^{+}+X\right) / \sigma\left(\nu_{l}+\mathcal{N} \rightarrow l^{-}+X\right)$ $(\mathcal{N}: P, N)$ as a function of $E_{\nu}$ in the range $10^{14} \mathrm{eV} \leq E_{\nu} \leq 10^{17} \mathrm{eV}$, with $E_{\mathcal{N}}=m_{\mathcal{N}}$. We have gotten $\sigma\left(\nu_{l}+P \rightarrow l^{-}+W^{+}+X\right) / \sigma\left(\nu_{l}+P \rightarrow l^{-}+X\right)=4.7 \times 10^{-3}$ and $\sigma\left(\nu_{l}+N \rightarrow\right.$ $\left.l^{-}+W^{+}+X\right) / \sigma\left(\nu_{l}+N \rightarrow l^{-}+X\right)=3.6 \times 10^{-3}$ for $E_{\nu}=10^{17} \mathrm{eV}$. 


\section{ACKNOWLEDGMENTS}

R. M. G-H. is grateful for the kind hospitality extended to her by the Abdus Salam International Centre for Theoretical Physics (Trieste, Italy) during summer stays in 2002 and 2003, when part of this work was made. She also thanks CONACyT (México) and VIEP-BUAP (Puebla, México) for financial support. A. R. would like to acknowledge Sistema Nacional de Investigadores and CONACyT (México) for financial support. 


\section{APPENDIX A}

By setting all fermion masses to zero, in this appendix we give the expressions for the quantities $T^{r, r^{\prime}}$, which are defined as follows:

$$
T_{P_{q}, P_{l}}^{r, r^{\prime}}=\mathcal{H}_{\mu \mu^{\prime}}^{P_{q}} \mathcal{F}_{r}^{\mu \nu}\left(\mathcal{F}_{r^{\prime}}^{\mu^{\prime} \nu^{\prime}}\right)^{*} \mathcal{L}_{\nu \nu^{\prime}}^{P_{l}}, \quad\left(r, r^{\prime}=1,2,3\right)
$$

with

$$
\begin{aligned}
\mathcal{L}_{\mu \mu^{\prime}}^{L, R} & =2\left\{p_{\mu} p_{\mu^{\prime}}^{\prime}+p_{\mu}^{\prime} p_{\mu^{\prime}}-g_{\mu \mu^{\prime}} p p^{\prime} \mp i \varepsilon_{\mu \mu^{\prime} \rho \sigma} p^{\rho} p^{\prime \sigma}\right\} \\
\mathcal{H}_{\mu \mu^{\prime}}^{L, R} & =2\left\{q_{\mu} q_{\mu^{\prime}}^{\prime}+q_{\mu}^{\prime} q_{\mu^{\prime}}-g_{\mu \mu^{\prime}} q q^{\prime} \mp i \varepsilon_{\mu \mu^{\prime} \rho \sigma} q^{\rho} q^{\prime \sigma}\right\} \\
\mathcal{F}_{1}^{\mu \nu} & =g^{\mu \nu} \\
\mathcal{F}_{2}^{\mu \nu} & =\varepsilon^{\mu} k^{\nu}-\varepsilon^{\nu} k^{\mu} \\
\mathcal{F}_{3}^{\mu \nu} & =i \varepsilon^{\mu \nu \rho \sigma} \varepsilon_{\rho} k_{\sigma}
\end{aligned}
$$

and $P_{q}, P_{l}$ being the polarization of the initial quark and initial neutrino, respectively. In general, these quantities are functions of scalar products of the momenta $p, q, p^{\prime}, q^{\prime}, k$ and the polarization vector $\varepsilon$ of the produced boson.

Using the polarization sum for the massive vector boson

$$
\sum_{\lambda} \varepsilon_{\mu}(k, \lambda) \varepsilon_{\nu}(k, \lambda)=-g_{\mu \nu}+\frac{k_{\mu} k_{\nu}}{M_{B}^{2}} .
$$

and the definitions given in (18) we get

$$
\begin{aligned}
& \sum_{\lambda} \mathcal{F}_{2}^{\mu \nu}\left(\mathcal{F}_{2}^{\rho \sigma}\right)^{*}=-g^{\mu \rho} k^{\nu} k^{\sigma}+g^{\mu \sigma} k^{\nu} k^{\rho}+g^{\nu \rho} k^{\mu} k^{\sigma}-g^{\nu \sigma} k^{\mu} k^{\rho} \\
& \sum_{\lambda} \mathcal{F}_{2}^{\mu \nu}\left(\mathcal{F}_{3}^{\rho \sigma}\right)^{*}=i\left(\varepsilon^{\mu \rho \sigma \beta} k^{\nu} k_{\beta}-\varepsilon^{\nu \rho \sigma \beta} k^{\mu} k_{\beta}\right) \\
& \sum_{\lambda} \mathcal{F}_{3}^{\mu \nu}\left(\mathcal{F}_{3}^{\rho \sigma}\right)^{*}=\left(g^{\mu \rho} g^{\nu \sigma}-g^{\mu \sigma} g^{\nu \rho}\right) M_{B}^{2}+\sum_{\lambda} \mathcal{F}_{2}^{\mu \nu}\left(\mathcal{F}_{2}^{\rho \sigma}\right)^{*}
\end{aligned}
$$

Aided by these expressions we obtain

$$
\sum_{\lambda} T^{22}=\left\{\begin{aligned}
8\left[-M_{B}^{2}\left(p p^{\prime} \cdot q q^{\prime}+p^{\prime} q^{\prime} \cdot p q-p^{\prime} q \cdot q^{\prime} p\right)\right. & \\
& \left.+2\left(p q \cdot k p^{\prime} \cdot k q^{\prime}+p^{\prime} q^{\prime} \cdot k p \cdot k q\right)\right] \\
8\left[-M_{B}^{2}\left(p p^{\prime} \cdot q q^{\prime}-p^{\prime} q^{\prime} \cdot p q+p^{\prime} q \cdot q^{\prime} p\right)\right. & \\
& \left.+2\left(p q^{\prime} \cdot k p^{\prime} \cdot k q+p^{\prime} q \cdot k p \cdot k q^{\prime}\right)\right]
\end{aligned}\right.
$$




$$
\begin{aligned}
\sum_{\lambda} R e T^{23}=\left\{\begin{array}{lr} 
\pm 16\left(-p q \cdot k p^{\prime} \cdot k q^{\prime}+p^{\prime} q^{\prime} \cdot k p \cdot k q\right) & L L, R R \\
\mp 16\left(p^{\prime} q \cdot k p \cdot k q^{\prime}-p q^{\prime} \cdot k p^{\prime} \cdot k q\right) & L R, R L
\end{array}\right. \\
\sum_{\lambda} T^{33}=\left\{\begin{array}{cc}
8\left[M_{B}^{2}\left(p p^{\prime} \cdot q q^{\prime}-p^{\prime} q \cdot p q^{\prime}-p q \cdot p^{\prime} q^{\prime}\right)\right. & L L, R R \\
\left.+2\left(p q \cdot k p^{\prime} \cdot k q^{\prime}+p^{\prime} q^{\prime} \cdot k p \cdot k q\right)\right] & \\
8\left[M_{B}^{2}\left(p p^{\prime} \cdot q q^{\prime}-p^{\prime} q \cdot p q^{\prime}-p q \cdot p^{\prime} q^{\prime}\right)\right. & L R, R L \\
\left.+2\left(p q^{\prime} \cdot k p^{\prime} \cdot k q+p^{\prime} q \cdot k p \cdot k q^{\prime}\right)\right] &
\end{array}\right.
\end{aligned}
$$

The remaining $T$, s can be expressed as follows

$$
\begin{aligned}
T^{11}= \begin{cases}16 p^{\prime} q^{\prime} \cdot p q & L L, R R \\
16 p^{\prime} q \cdot p q^{\prime} & L R, R L\end{cases} \\
\operatorname{Re} T^{12}=\left\{\begin{array}{cc}
-8\left(\varepsilon p \cdot p^{\prime} q^{\prime} \cdot q k+\varepsilon p^{\prime} \cdot p q \cdot q^{\prime} k\right. & L L, R R \\
\left.-\varepsilon q \cdot p^{\prime} q^{\prime} \cdot p k-\varepsilon q^{\prime} \cdot p q \cdot p^{\prime} k\right) & \\
\operatorname{Re} T^{13}=\left\{\begin{array}{c}
-8 p \cdot p^{\prime} q \cdot q^{\prime} k+\varepsilon p^{\prime} \cdot p q^{\prime} \cdot q k \\
-\varepsilon q \cdot p q^{\prime} \cdot p^{\prime} k-\varepsilon q^{\prime} \cdot p^{\prime} q \cdot p k \\
\mp 8\left(\varepsilon p \cdot p^{\prime} q^{\prime} \cdot q k-\varepsilon p^{\prime} \cdot p q \cdot q^{\prime} k\right.
\end{array}\right. & L L, R R \\
\left.\quad+\varepsilon q^{\prime} \cdot p q \cdot p^{\prime} k-\varepsilon q \cdot p^{\prime} q^{\prime} \cdot p k\right) \\
\pm 8\left(\varepsilon p \cdot p^{\prime} q \cdot q^{\prime} k-\varepsilon p^{\prime} \cdot p q^{\prime} \cdot q k\right.
\end{array}\right. \\
\left.-\varepsilon q^{\prime} \cdot p^{\prime} q \cdot p k+\varepsilon q \cdot p q^{\prime} \cdot p^{\prime} k\right)
\end{aligned}
$$

Using (A1) and (18) we get

$$
\begin{aligned}
& \sum_{\lambda} \varepsilon P_{1} \cdot \varepsilon P_{2}=-P_{1} P_{2}+\frac{k P_{1} \cdot k P_{2}}{M_{B}^{2}} \\
& \sum_{\lambda} \varepsilon P \cdot \mathcal{F}_{2}^{\mu \nu}=\mathcal{F}_{2}^{\mu \nu}(\varepsilon \rightarrow-P) \\
& \sum_{\lambda} \varepsilon P \cdot \mathcal{F}_{3}^{\mu \nu}=\mathcal{F}_{3}^{\mu \nu}(\varepsilon \rightarrow-P)
\end{aligned}
$$

for $P, P_{1}, P_{2}=p, q, p^{\prime}, q^{\prime}$. Hence

$$
\sum_{\lambda} \varepsilon P_{1} \cdot \varepsilon P_{2} T^{11}=\left(-P_{1} P_{2}+\frac{k P_{1} \cdot k P_{2}}{M_{B}^{2}}\right) T^{11}
$$




$$
\begin{aligned}
& \sum_{\lambda} \varepsilon P \cdot \operatorname{Re} T^{12}=\operatorname{Re} T^{12}(\varepsilon \rightarrow-P) \\
& \sum_{\lambda} \varepsilon P \cdot \operatorname{Re} T^{13}=\operatorname{Re} T^{13}(\varepsilon \rightarrow-P)
\end{aligned}
$$




\section{Figure Captions}

Fig. 1: Feynman diagrams for heavy boson production from the initial (a) and final (b) lepton, from the initial (c) and final (d) quark and via the non-Abelian couplings (e).

Figs. 2.1 - 2.5: Feynman diagrams for processes (P.1) - (P.5): boson production from the incoming neutrino (a), the outgoing lepton (b), the initial (c) and final (d) quark, and through the non-Abelian couplings (e). u stands for $u, c, \bar{d}, \bar{s}, \bar{b}$; $d$ for $d, s, b, \bar{u}, \bar{c}$.

Fig. 3: $\sigma\left(\nu_{l}+P \rightarrow l^{-}+W^{+}+X\right)$ as a function of $E_{\nu}$ in the range $10^{14} \mathrm{eV} \leq E_{\nu} \leq 10^{17} \mathrm{eV}$, with $E_{P}=m_{P}$. Taking $W_{c}=10 \mathrm{GeV}^{2}$ and a) $Q_{c}^{2}=Q_{c}^{\prime 2}=2 G e V^{2}$ (curve A), b) $Q_{c}^{2}=Q_{c}^{\prime 2}=$ $4 \mathrm{GeV}^{2}$ (curve B), c) $Q_{c}^{2}=Q_{c}^{\prime 2}=6 \mathrm{GeV}^{2}$ (curve C), d) $Q_{c}^{2}=Q_{c}^{\prime 2}=8 \mathrm{GeV}^{2}$ (curve D).

Fig. 4: $\sigma\left(\nu_{l}+P \rightarrow l^{-}+W^{+}+X\right)$ and $\sigma\left(\nu_{l}+P \rightarrow l^{-}+X\right)$ as a function of $E_{\nu}$ in the range $10^{14} \mathrm{eV} \leq E_{\nu} \leq 10^{17} \mathrm{eV}$, with $E_{P}=m_{P}$. Taking $W_{c}=10 \mathrm{GeV}^{2}$ and $Q_{c}^{2}=Q_{c}^{\prime 2}=4 \mathrm{GeV}^{2}$.

Fig. 5: $\sigma\left(\nu_{l}+N \rightarrow l^{-}+W^{+}+X\right)$ and $\sigma\left(\nu_{l}+N \rightarrow l^{-}+X\right)$ as a function of $E_{\nu}$ in the range $10^{14} \mathrm{eV} \leq E_{\nu} \leq 10^{17} \mathrm{eV}$, with $E_{N}=m_{N}$. Taking $W_{c}=10 \mathrm{GeV}^{2}$ and $Q_{c}^{2}=Q_{c}^{\prime 2}=4 \mathrm{GeV}^{2}$.

Fig. 6: $\sigma\left(\nu_{l}+\mathcal{N} \rightarrow l^{-}+W^{+}+X\right) / \sigma\left(\nu_{l}+\mathcal{N} \rightarrow l^{-}+X\right)(\mathcal{N}: P, N)$ as a function of $E_{\nu}$ in the range $10^{14} \mathrm{eV} \leq E_{\nu} \leq 10^{17} \mathrm{eV}$, with $E_{\mathcal{N}}=m_{\mathcal{N}}$. Taking $W_{c}=10 \mathrm{GeV}^{2}$ and $Q_{c}^{2}=Q_{c}^{\prime 2}=4 \mathrm{GeV}^{2}$. 


\section{REFERENCES}

[1] AMANDA Collaboration, E. Andres et al., Nature 410, 441 (2001); ANTARES Collaboration, Y. Becherini et al., e-Print Archive: hep-ph/0211173; AUGER Collaboration, D. Zavrtanik et al., Nucl. Phys. Proc. Suppl. 85, 324 (2002); NESTOR Collaboration, P. K. F. Grieder et al., Nuovo Cim. 24C, 771 (2001); RICE Collaboration, I. Kravchenko et al., Astropart. Phys. 19, 15 (2003).

[2] V. S. Berezinsky, Nucl. Phys. B28A, 352 (1992); V. S. Berezinsky and G. Zatsepin, Proceedings of the 1976 DUMAND Summer Workshop (A. Roberts and R. Donaldson, eds.), p. 215 (1977); T. Stanev, Nucl. Phys. B14A, 17 (1990); K. Greisen, Phys. Rev. Lett. 16, 748 (1966); C. T. Hill and D. N. Schramm, Phys. Lett. B131, 247 (1983); and Phys. Rev. D31, 564 (1985); R. Gandhi et al., Phys. Rev. D58, 093009 (1998) and references therein.

[3] R. Gandhi et al., Phys. Rev. D58, 093009 (1998).

[4] H. H. Chen, Phys. Rev. 1, 3197 (1970).

[5] J. Reiff, Nucl. Phys. B23, 387 (1970); and Nucl. Phys. B28, 495 (1971).

[6] R. W. Brown and J. Smith, Phys. Rev. 3, 207 (1971); R. W. Brown, R. H. Hobbs and J. Smith, Phys. Rev. 4, 794 (1971); R. W. Brown, R. H. Hobbs, J. Smith and N. Stanko, Phys. Rev. 6, 3273 (1972).

[7] F. A. Berends and G. B. West, Phys. Rev. 3, 262 (1971).

[8] S. L. Glashow, Nucl. Phys. 22, 579 (1961); S. Weinberg, Phys. Rev. Lett. 19, 1264 (1967); A. Salam, Proc. 8th NOBEL Symposium, ed. N. Svartholm (Almqvist and Wiksell, Stockholm, 1968), p. 367.

[9] R. P. Feynman: Photon-hadron interactions. Reading: Benjamin 1972.

[10] Z. Fodor et al., Phys. Lett. B561, 191 (2003); T. Han and D. Hooper, e-Print Archive: 
hep-ph/0307120.

[11] J. Pumplin et al., JHEP 207, 12 (2002); D. Stump et al., e-Print Archive: hep$\mathrm{ph} / 0303013$.

[12] E. Byckling and Kajantie: Particle kinematics. New York: Willey 1972.

[13] Particle Data Group, K. Hagiwara et al., Phys. Rev. D66, 010001 (2002). 


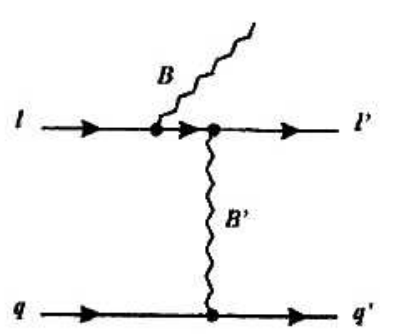

(a)

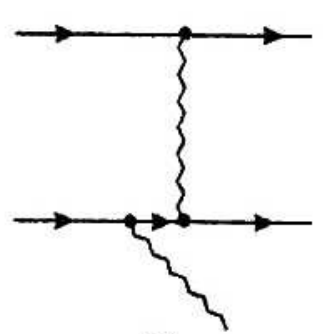

(c)

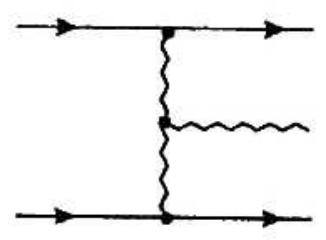

(e)

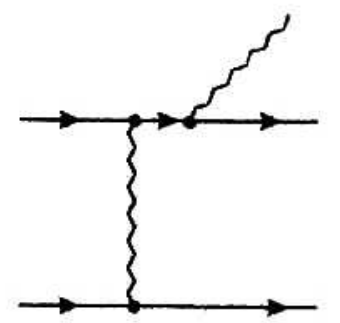

(b)

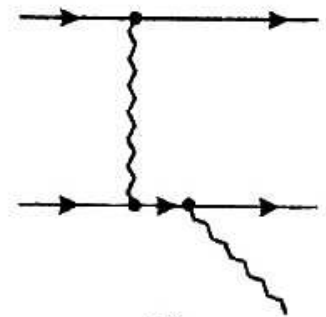

(d)

Fig. 1 
(2.1)
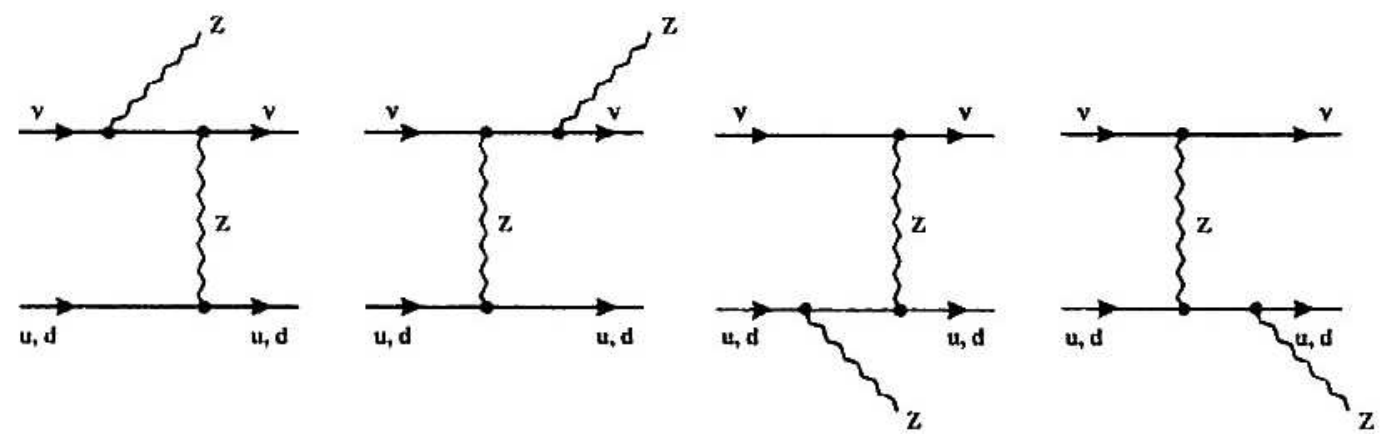

(2.2)
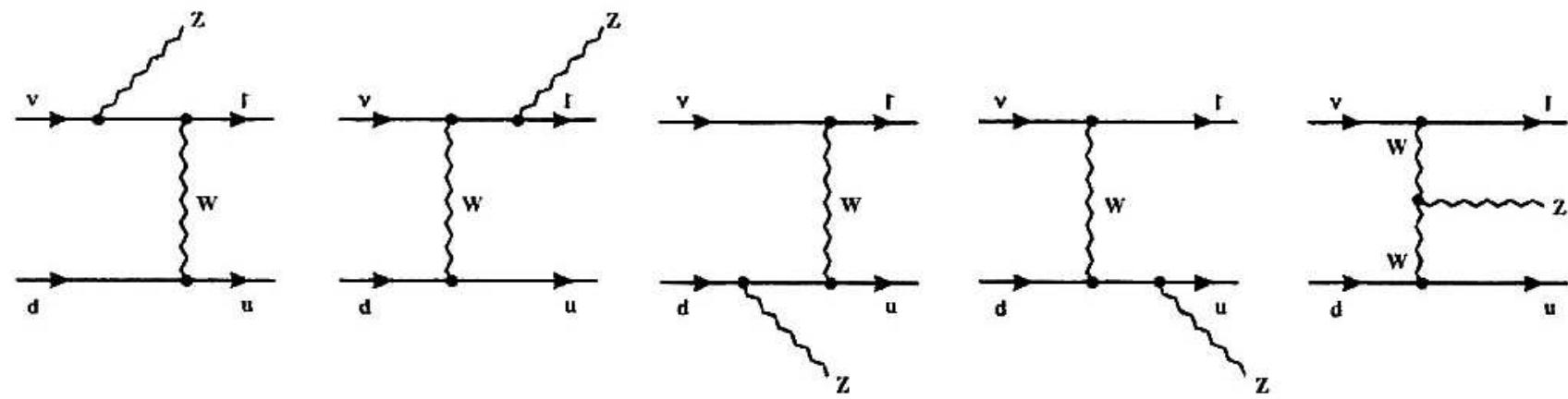

(2.3)
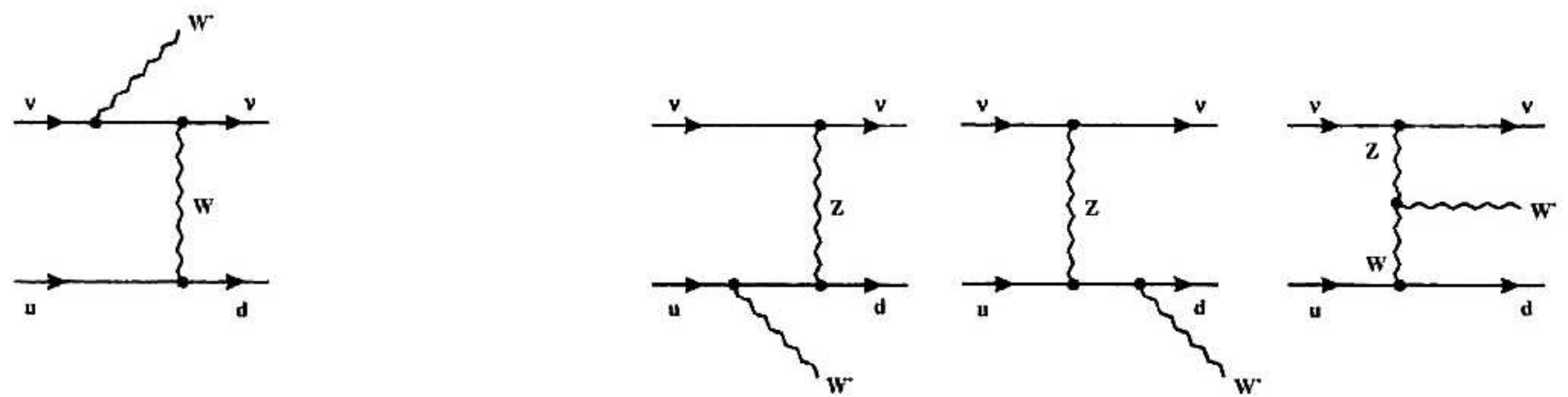

(2.4)
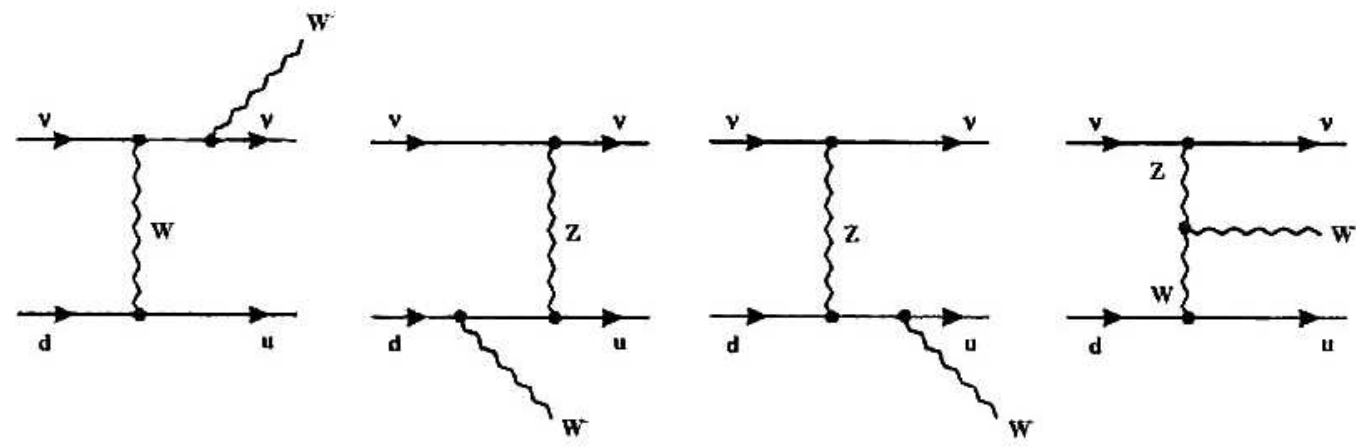

(2.5)

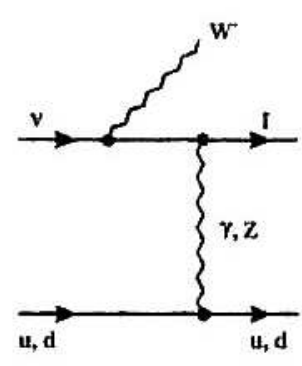

(a)

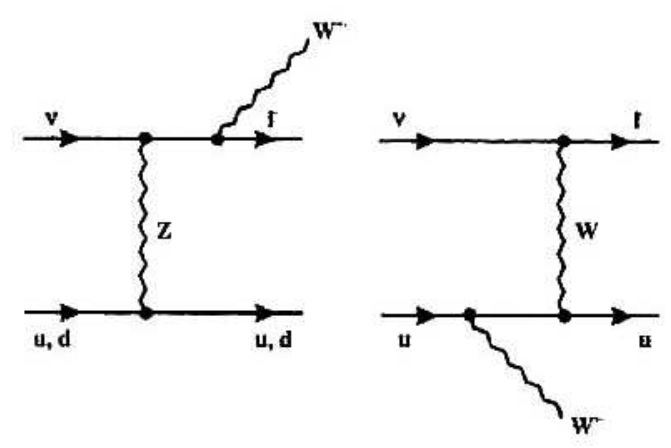

(b)

(c)

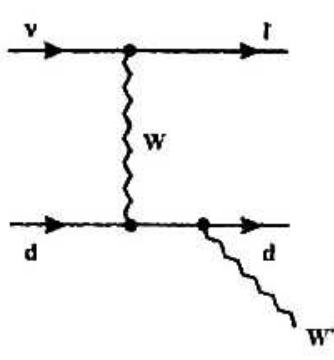

(d)

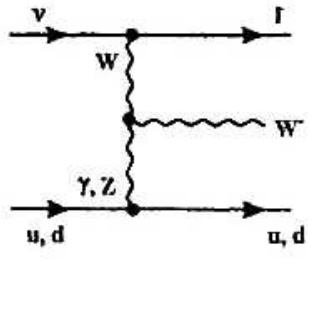

(e)

Fig. 2 


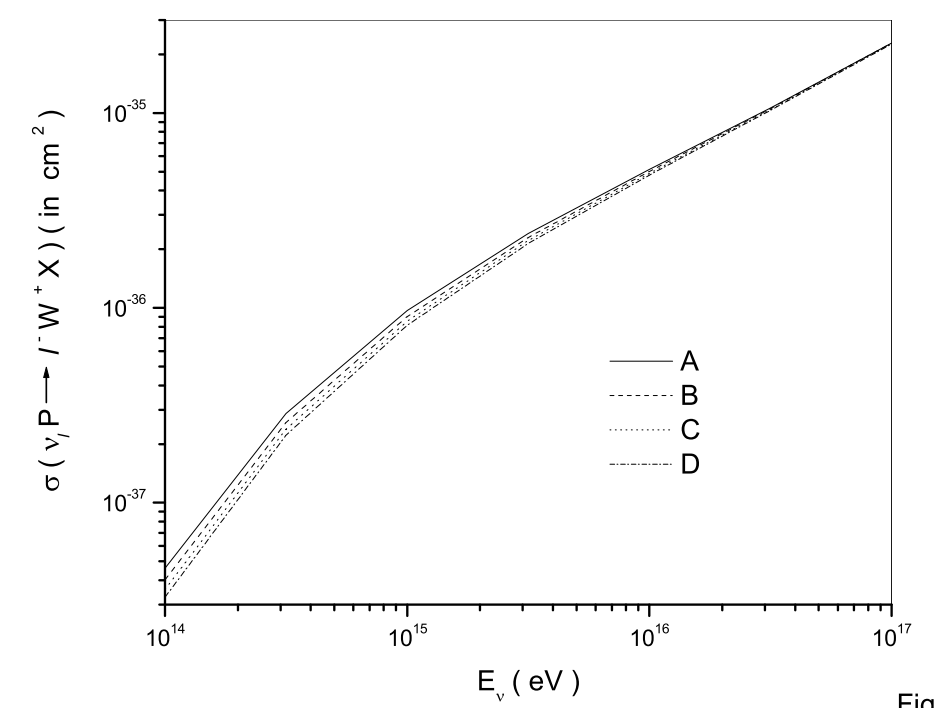

Fig. 3 


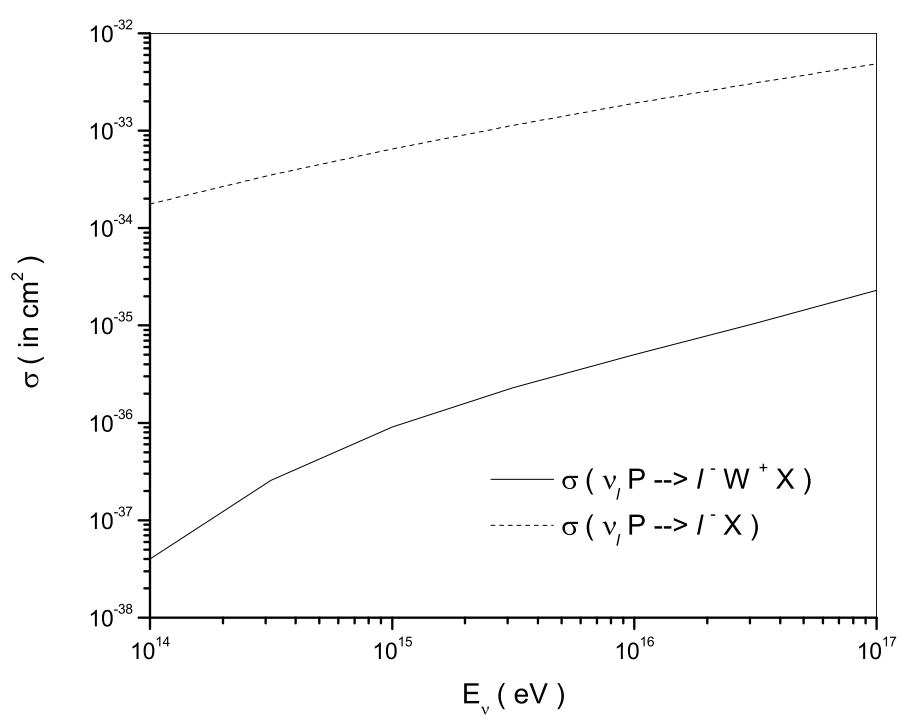

Fig. 4 


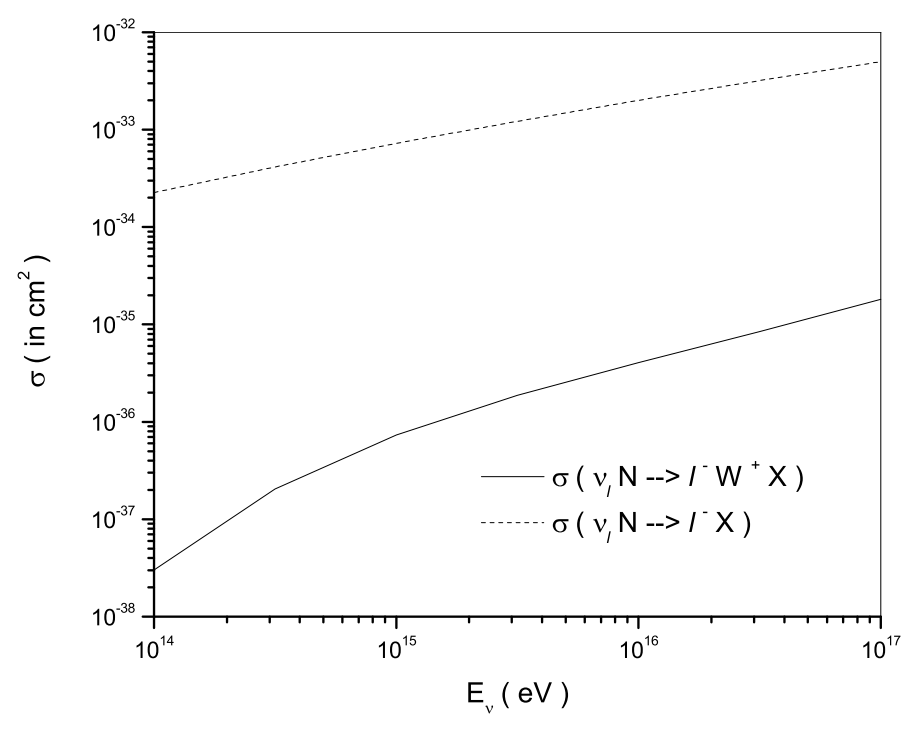

Fig. 5 


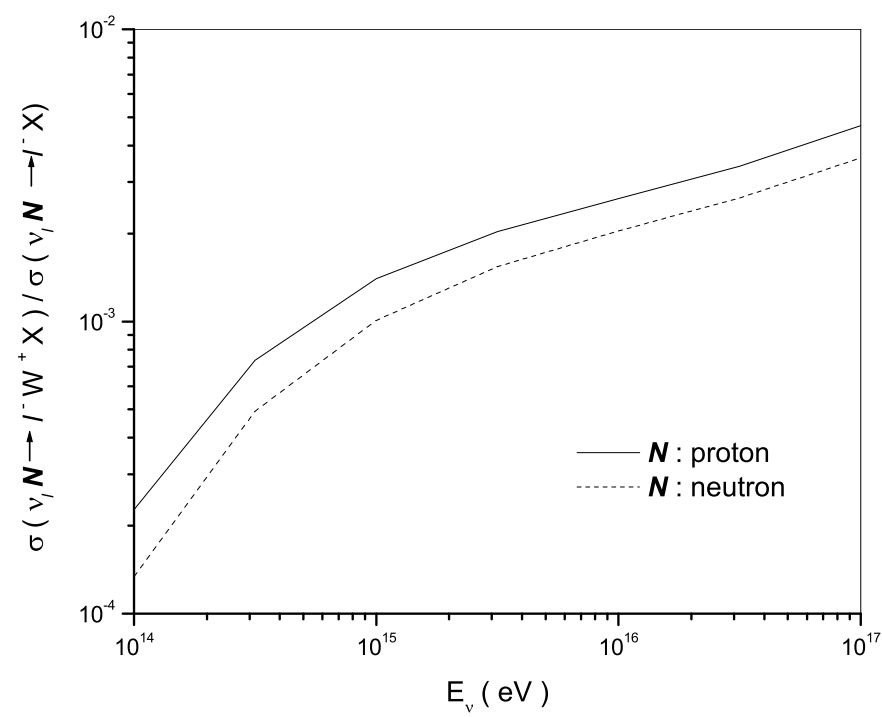

Fig. 6 